Proc. Indian Acad. Sci. (Math. Sci.) Vol. 113, No. 2, May 2003, pp. 183-187.

Printed in India

\title{
Fixed point of multivalued mapping in uniform spaces
}

\author{
DURAN TÜRKOGLU and BRIAN FISHER* \\ Department of Mathematics, Faculty of Science and Arts, University of Kirikkale, \\ 71450-Yahsihan Kirikkale, Turkey \\ *Department of Mathematical Sciences, Faculty of Computer Sciences and \\ Engineering, De Montfort University, The Gateway, Leicester, LE1 9BH, England \\ E-mail: duran_t@hotmail.com; fbr@le.ac.uk
}

MS received 31 December 2001; revised 10 July 2002

Abstract. In this paper we prove some new fixed point theorems for multivalued mappings on orbitally complete uniform spaces.

Keywords. Fixed point; multivalued mappings; orbitally complete; uniform space.

\section{Introduction}

Let $(X, \mathscr{U})$ be a uniform space. A family $\left\{d_{i}: i \in I\right\}$ of pseudometrics on $X$ with indexing set $I$, is called an associated family for the uniformity $\mathscr{U}$ if the family

$$
\beta=\{V(i, \varepsilon): i \in I ; \varepsilon>0\},
$$

where

$$
V(i, \varepsilon)=\left\{(x, y): x, y \in X, d_{i}(x, y)<\varepsilon\right\}
$$

is a sub-base for the uniformity $\mathscr{U}$. We may assume that $\beta$ itself is a base by adjoining finite intersection of members of $\beta$, if necessary. The corresponding family of pseudometrics is called an associated family for $\mathscr{U}$. An associated family for $\mathscr{U}$ will be denoted by $p^{*}$. For details the reader is referred to [1345678].

Let $A$ be a nonempty subset of a uniform space $X$. Define

$$
\Delta^{*}(A)=\sup \left\{d_{i}(x, y): x, y \in A, i \in I\right\},
$$

where

$$
\left\{d_{i}: i \in I\right\}=p^{*} .
$$

Then $\Delta^{*}$ is called an augmented diameter of $A$. Further, $A$ is said to be $p^{*}$-bounded if $\Delta^{*}(A)<\infty$. Let

$$
2^{X}=\left\{A: A \text { is a nonempty, closed and } p^{*} \text { - bounded subset of } X\right\} .
$$

For any nonempty subsets $A$ and $B$ of $X$, define

$$
d_{i}(x, A)=\inf \left\{d_{i}(x, a): a \in A\right\}, i \in I
$$




$$
\begin{aligned}
H_{i}(A, B) & =\max \left\{\sup _{a \in A} d_{i}(a, B), \sup _{b \in B} d_{i}(A, b)\right\} \\
& =\sup _{x \in X}\left\{\left|d_{i}(x, A)-d_{i}(x, B)\right|\right\} .
\end{aligned}
$$

It is well-known that on $2^{X}, H_{i}$ is a pseudometric, called the Hausdorff pseudometric induced by $d_{i}, i \in I$.

Let $(X, \mathscr{U})$ be a uniform space with an augmented associated family $p^{*} . p^{*}$ also induces a uniformity $\mathscr{U}^{*}$ on $2^{X}$ defined by the base

$$
\beta^{*}=\left\{V^{*}(i, \varepsilon): i \in I, \varepsilon>0\right\}
$$

where

$$
V^{*}(i, \varepsilon)=\left\{(A, B): A, B \in 2^{X}, H_{i}(A, B)<\varepsilon\right\} .
$$

The space $\left(2^{X}, \mathscr{U}^{*}\right)$ is a uniform space called the hyperspace of $(X, \mathscr{U})$.

\section{DEFINITION 1.}

The collection of all filters on a given set $X$ is denoted by $\Phi(X)$. An order relation is defined on $\Phi(X)$ by the rule $\mathscr{F}_{1}<\mathscr{F}_{2}$ iff $\mathscr{F}_{1} \supset \mathscr{F}_{2}$. If $\mathscr{F}^{*}<\mathscr{F}$, then $\mathscr{F}^{*}$ is called a subfilter of $\mathscr{F}$.

\section{DEFINITION 2.}

Let $(X, \mathscr{U})$ be a uniform space defined by $\left\{d_{i}: i \in I\right\}=p^{*}$. If $F: X \rightarrow 2^{X}$ is a multivalued mapping, then

(i) $x \in X$ is called a fixed point of $F$ if $x \in F x$;

(ii) An orbit of $F$ at a point $x_{0} \in X$ is a sequence $\left\{x_{n}\right\}$ given by

$$
O\left(F, x_{0}\right)=\left\{x_{n}: x_{n} \in F x_{n-1}, n=1,2, \ldots\right\}
$$

(iii) A uniform space $X$ is called $F$-orbitally complete if every Cauchy filter which is a subfilter of an orbit of $F$ at each $x \in X$ converges to a point of $X$.

\section{DEFINITION 3.}

Let $(X, \mathscr{U})$ be a uniform space and let $F: X \rightarrow X$ be a mapping. A single-valued mapping $F$ is orbitally continuous if $\lim \left(T^{n_{i}} x\right)=u$ implies $\lim T\left(T^{n_{i}} x\right)=T u$ for each $x \in X$.

\section{Main results}

Theorem 1. Let $(X, \mathscr{U})$ be an $F$-orbitally complete Hausdorff uniform space defined by $\left\{d_{i}: i \in I\right\}=p^{*}$ and $\left(2^{X}, \mathscr{U}^{*}\right)$ a hyperspace and let $F: X \rightarrow 2^{X}$ be a continuous mapping with $F x$ compact for each $x$ in $X$. Assume that

$$
\begin{aligned}
\min \{ & \left.H_{i}(F x, F y)^{r}, d_{i}(x, F x) d_{i}(y, F y)^{r-1}, d_{i}(y, F y)^{r}\right\} \\
& +a_{i} \min \left\{d_{i}(x, F y), d_{i}(y, F x)\right\} \leq\left[b_{i} d_{i}(x, F x)\right. \\
& \left.+c_{i} d_{i}(x, y)\right] d_{i}(y, F y)^{r-1}
\end{aligned}
$$

for all $i \in I$ and $x, y \in X$, where $r \geq 1$ is an integer, $a_{i}, b_{i}, c_{i}$ are real numbers such that $0<b_{i}+c_{i}<1$, then $F$ has a fixed point. 
Proof. Let $x_{0}$ be an arbitrary point in $X$ and consider the sequence $\left\{x_{n}\right\}$ defined by

$$
x_{1} \in F x_{0}, x_{2} \in F x_{1}, \ldots, x_{n} \in F x_{n-1}, \ldots
$$

Let us suppose that $d_{i}\left(x_{n}, F x_{n}\right)>0$ for each $i \in I$ and $n=0,1,2, \ldots$ (Otherwise for some positive integer $n, x_{n} \in F x_{n}$ as desired.)

Let $U \in \mathscr{U}$ be an arbitrary entourage. Since $\beta$ is a base for $\mathscr{U}$, there exists $V(i, \varepsilon) \in \beta$ such that $V(i, \varepsilon) \subseteq U$. Now $y \rightarrow d_{i}\left(x_{0}, y\right)$ is continuous on the compact set $F x_{0}$ and this implies that there exists $x_{1} \in F x_{0}$ such that $d_{i}\left(x_{0}, x_{1}\right)=d_{i}\left(x_{0}, F x_{0}\right)$. Similarly, $F x_{1}$ is compact so there exists $x_{2} \in F x_{1}$ such that $d_{i}\left(x_{1}, x_{2}\right)=d_{i}\left(x_{1}, F x_{1}\right)$. Continuing, we obtain a sequence $\left\{x_{n}\right\}$ such that $x_{n+1} \in F x_{n}$ and $d_{i}\left(x_{n}, x_{n+1}\right)=d_{i}\left(x_{n}, F x_{n}\right)$.

For $x=x_{n-1}$, and $y=x_{n}$ by condition (1) we have

$$
\begin{aligned}
\min \{ & \left.H_{i}\left(F x_{n-1}, F x_{n}\right)^{r}, d_{i}\left(x_{n-1}, F x_{n-1}\right) d_{i}\left(x_{n}, F x_{n}\right)^{r-1}, d_{i}\left(x_{n}, F x_{n}\right)^{r}\right\} \\
& +a_{i} \min \left\{d_{i}\left(x_{n-1}, F x_{n}\right), d_{i}\left(x_{n}, F x_{n-1}\right)\right\} \leq\left[b_{i} d_{i}\left(x_{n-1}, F x_{n-1}\right)\right. \\
& \left.+c_{i} d_{i}\left(x_{n-1}, x_{n}\right)\right] d_{i}\left(x_{n}, F x_{n}\right)^{r-1}
\end{aligned}
$$

or since $d_{i}\left(x_{n}, F x_{n-1}\right)=0, x_{n} \in F x_{n-1}$. Hence we have

$$
\begin{aligned}
& \min \left\{d_{i}\left(x_{n}, x_{n+1}\right)^{r}, d_{i}\left(x_{n-1}, x_{n}\right) d_{i}\left(x_{n}, x_{n+1}\right)^{r-1}\right\} \\
& \leq\left[b_{i} d_{i}\left(x_{n-1}, x_{n}\right)+c_{i} d_{i}\left(x_{n-1}, x_{n}\right)\right] d_{i}\left(x_{n}, x_{n+1}\right)^{r-1}
\end{aligned}
$$

and it follows that

$$
\begin{gathered}
\min \left\{d_{i}\left(x_{n}, x_{n+1}\right)^{r}, d_{i}\left(x_{n-1}, x_{n}\right) d_{i}\left(x_{n}, x_{n+1}\right)^{r-1}\right\} \\
\leq\left(b_{i}+c_{i}\right) d_{i}\left(x_{n-1}, x_{n}\right) d_{i}\left(x_{n}, x_{n+1}\right)^{r-1} .
\end{gathered}
$$

Since

$$
d_{i}\left(x_{n-1}, x_{n}\right) d_{i}\left(x_{n}, x_{n+1}\right)^{r-1} \leq\left(b_{i}+c_{i}\right) d_{i}\left(x_{n-1}, x_{n}\right) d_{i}\left(x_{n}, x_{n+1}\right)^{r-1}
$$

is not possible (as $0<b_{i}+c_{i}<1$ ), we have

$$
d_{i}\left(x_{n}, x_{n+1}\right)^{r} \leq\left(b_{i}+c_{i}\right) d_{i}\left(x_{n-1}, x_{n}\right) d_{i}\left(x_{n}, x_{n+1}\right)^{r-1}
$$

or

$$
d_{i}\left(x_{n}, x_{n+1}\right)^{r} \leq k_{i} d_{i}\left(x_{n-1}, x_{n}\right) d_{i}\left(x_{n}, x_{n+1}\right)^{r-1}
$$

where $k_{i}=b_{i}+c_{i}, 0<k_{i}<1$.

Proceeding in this manner we get

$$
\begin{gathered}
d_{i}\left(x_{n}, x_{n+1}\right) \leq k_{i} d_{i}\left(x_{n-1}, x_{n}\right) \\
\leq k_{i}^{2} d_{i}\left(x_{n-2}, x_{n-1}\right) \\
\quad \vdots \\
\leq k_{i}^{n} d_{i}\left(x_{0}, x_{1}\right)
\end{gathered}
$$


Hence we obtain

$$
\begin{aligned}
d_{i}\left(x_{n}, x_{m}\right) & \leq d_{i}\left(x_{n}, x_{n+1}\right)+d_{i}\left(x_{n+1}, x_{n+2}\right)+\cdots+d_{i}\left(x_{m-1}, x_{m}\right) \\
& \leq\left(k_{i}^{n}+k_{i}^{n+1}+\cdots+k_{i}^{m-1}\right) d_{i}\left(x_{0}, x_{1}\right) \\
& \leq k_{i}^{n}\left(1+k_{i}+\cdots+k_{i}^{m-n-1}\right) d_{i}\left(x_{0}, x_{1}\right) \\
& \leq \frac{k_{i}^{n}}{1-k} d_{i}\left(x_{0}, x_{1}\right) .
\end{aligned}
$$

Since $\lim _{n \rightarrow \infty} k_{i}^{n}=0$, it follows that there exists $N(i, \varepsilon)$ such that $d_{i}\left(x_{n}, x_{m}\right)<\varepsilon$ and hence $\left(x_{n}, x_{m}\right) \in U$ for all $n, m \geq N(i, \varepsilon)$. Therefore the sequence $\left\{x_{n}\right\}$ is a Cauchy sequence in the $d_{i}$-uniformity on $X$.

Let $S_{p}=\left\{x_{n}: n \geq p\right\}$ for all positive integers $p$ and let $\beta$ be the filter basis $\left\{S_{p}: p=1,2, \ldots\right\}$. Then since $\left\{x_{n}\right\}$ is a $d_{i}$-Cauchy sequence for each $i \in I$, it is easy to see that the filter basis $\beta$ is a Cauchy filter in the uniform space $(X, \mathscr{U})$. To see this we first note that the family $\{V(i, \varepsilon): i \in I\}$ is a base for $\mathscr{U}$ as $p^{*}=\left\{d_{i}: i \in I\right\}$. Now since $\left\{x_{n}\right\}$ is a $d_{i}$-Cauchy sequence in $X$, there exists a positive integer $p$ such that $d_{i}\left(x_{n}, x_{m}\right)<\varepsilon$ for $m \geq p, n \geq p$. This implies that $S_{p} \times S_{p} \subseteq V(i, \varepsilon)$. Thus given any $U \in \mathscr{U}$, we can find an $S_{p} \in \beta$ such that $S_{p} \times S_{p} \subset U$. Hence $\beta$ is a Cauchy filter in $(X, \mathscr{U})$. Since $(X, \mathscr{U})$ is $F$-orbitally complete and Hausdorff space, $S_{p} \rightarrow z$ for some $z \in X$. Consequently $F\left(S_{p}\right) \rightarrow F z$ (follows from the continuity of $F$ ). Also

$$
S_{p+1} \subseteq F\left(S_{p}\right)=\cup\left\{F x_{n}: n \geq p\right\}
$$

for $p=1,2, \ldots$ It follows that $z \in F z$. Hence $z$ is a fixed point of $F$. This completes the proof.

If we take $r=1$ in Theorem 1, then we obtain the following theorem.

Theorem 2. Let $(X, \mathscr{U})$ be an $F$-orbitally complete Hausdorff uniform space defined by $\left\{d_{i}: i \in I\right\}=p^{*}$ and $\left(2^{X}, \mathscr{U}^{*}\right)$ a hyperspace, let $F: X \rightarrow 2^{X}$ be a continuous mapping and $F x$ compact for each $x$ in $X$. Assume that

$$
\begin{aligned}
\min & \left\{H_{i}(F x, F y), d_{i}(x, F x), d_{i}(y, F y)\right\} \\
& +a_{i} \min \left\{d_{i}(x, F y), d_{i}(y, F x)\right\} \leq b_{i} d_{i}(x, F x)+c_{i} d_{i}(x, y)
\end{aligned}
$$

for all $i \in I$ and $x, y \in X$, where $a_{i}, b_{i}, c_{i}$ are real numbers such that $0<b_{i}+c_{i}<1$, then $F$ has a fixed point.

We denote that if $F$ is a single valued mapping on $X$, then we can write $d_{i}(F x, F y)=$ $H_{i}(F x, F y), x, y \in X, i \in I$.

Thus we obtain the following theorem as a consequence of the Theorem 2 .

Theorem 3. Let $(X, \mathscr{U})$ be a $T$-orbitally complete Hausdorff uniform space and let $T$ : $X \rightarrow X$ be a $T$-orbitally continuous mapping satisfying

$$
\begin{aligned}
\min & \left\{d_{i}(T x, T y), d_{i}(x, T x), d_{i}(y, T y)\right\} \\
& +a_{i} \min \left\{d_{i}(x, T y), d_{i}(y, T x)\right\} \leq b_{i} d_{i}(x, T x)+c_{i} d_{i}(x, y)
\end{aligned}
$$

for all $x, y \in X, i \in I$ and $a_{i}, b_{i}, c_{i}$ are real numbers such that $0<b_{i}+c_{i}<1$. Then $T$ has a fixed point and which is unique whenever $a_{i}>c_{i}>0$. 
Proof. Define a mapping $F$ of $X$ into $2^{X}$ by putting $F x=\{T x\}$ for all $x$ in $X$. It follows that $F$ satisfies the conditions of Theorem 2 . Hence $T$ has a fixed point.

Now if $a_{i}>c_{i}>0$, we show that $T$ has a unique fixed point. Assume that $T$ has two fixed points $z$ and $w$ which are distinct. Since $d_{i}(z, T z)=0$ and $d_{i}(w, T w)=0$, then by the condition (2),

$$
a_{i} \min \left\{d_{i}(z, T w), d_{i}(w, T z)\right\} \leq c_{i} d_{i}(z, w)
$$

or

$$
\begin{aligned}
a_{i} d_{i}(z, w) & \leq c_{i} d_{i}(z, w), \\
d_{i}(z, w) & \leq \frac{c_{i}}{a_{i}} d_{i}(z, w)
\end{aligned}
$$

which is impossible. Thus if $a_{i}>c_{i}>0$, then $T$ has a unique fixed point in $X$. This completes the proof.

We note that if $a_{i}=-1$ in condition (3), then one gets the following result as a corollary.

\section{COROLLARY 4.}

Let $T$ be an orbitally cotinuous self-map of a $T$-orbitally complete uniform space $(X, \mathscr{U})$ satisfying the condition

$$
\begin{aligned}
& \min \left\{d_{i}(T x, T y), d_{i}(x, T x), d_{i}(y, T y)\right\} \\
& \quad-\min \left\{d_{i}(x, T y), d_{i}(y, T x)\right\} \leq b_{i} d_{i}(x, T x)+c_{i} d_{i}(x, y),
\end{aligned}
$$

$x, y \in X, i \in I$ and $0<b_{i}+c_{i}<1$. Then for each $x \in X$, the sequence $\left\{T^{n} x\right\}$ converges to a fixed point of $T$.

Remark 1 . If we replace the uniform space $(X, \mathscr{U})$ in Theorem 3 and Corollary 4 by a metric space (i.e. a metrizable uniform space), then Theorem 1 and Corollary 1 of Dhage [2] will follow as special cases of our results.

\section{Acknowledgement}

This research was supported by the Scientific and Technical Research Council of Turkey, TBAG-1742 (1999).

\section{References}

[1] Acharya S P, Some results on fixed point in uniform space, Yokohama Math. J. XXII (1) (1974) 105-116

[2] Dhage B C, Some results for the maps with a nonunique fixed point, Indian J. Pure Appl. Math. 16(3) (1985) 245-256

[3] Kelley J L, General Topology (Van Nonstrand Company Inc.) (1955)

[4] Mishra S N and Singh S N, Fixed point of multivalued mapping in uniform spaces, Bull. Cal. Math. Soc. 77 (1985) 323-329

[5] Taraftar E, An approach to fixed point theorems on uniform spaces, Trans. Amer. Math. Soc. 77 (1985) 209-225

[6] Taylor W W, Fixed point theorems for nonexpansive mappings in linear topological spaces, J. Math. Anal. Appl. 40 (1972) 164-173

[7] Thron W J, Topological structures (New York: Holt, Rinehart and Winston) (1966)

[8] Türkoglu D, Özer O and Fisher B, Some fixed point theorems for set valued mapping in uniform spaces, Demonstratio Math. 2 (1999) 395-400 